\title{
A new interpretation of Jensen's inequality and geometric properties of $\phi$-means
}

Yasuo Nakasuji ${ }^{1}$, Keisaku Kumahara ${ }^{1}$ and Sin-Ei Takahasi $2^{*^{*}}$

* Correspondence: sin-ei@emperor yz.yamagata-u.ac.jp

${ }^{2}$ Toho University, Yamagata

University (Professor Emeritus), Chiba 273-0866, Japan

Full list of author information is available at the end of the article

\section{Abstract}

We introduce a mean of a real-valued measurable function $f$ on a probability space induced by a strictly monotone function $\phi$. Such a mean is called a $\phi$-mean of $f$ and written by $M_{\phi}(f)$. We first give a new interpretation of Jensen's inequality by $\phi$-mean. Next, as an application, we consider some geometric properties of $M_{\phi}(f)$, for example, refinement, strictly monotone increasing (continuous) $\phi$-mean path, convexity, etc. Mathematics Subject Classification (2000): Primary 26E60; Secondary 26B25, 26 B05.

Keywords: Jensen's inequality, Mean, Refinement, Convexity, Concavity

\section{Introduction}

We are interested in means of real-valued measurable functions induced by strictly monotone functions. These means are somewhat different from continuously differentiable means, i.e., $C^{1}$-means introducing by Fujii et al. [1], but they include many known numerical means. Here we first give a new interpretation of Jensen's inequality by such a mean and we next consider some geometric properties of such means, as an application of it.

Throughout the paper, we denote by $(\Omega, \mu), I$ and $f$ a probability space, an interval of $\mathbb{R}$ and a real-valued measurable function on $\Omega$ with $f(\omega) \in I$ for almost all $\omega \in \Omega$, respectively. Let $C(I)$ be the real linear space of all continuous real-valued functions defined on $I$. Let $C_{s m}^{+}(I)$ (resp. $\left.C_{s m}^{-}(I)\right)$ be the set of all $\phi \in C(I)$ which is strictly monotone increasing (resp. decreasing) on $I$. Then $C_{s m}^{+}(I)$ (resp. $C_{s m}^{-}(I)$ ) is a positive (resp. negative) cone of $C(I)$. Put $C_{s m}(I)=C_{s m}^{+}(I) \cup C_{s m}^{-}(I)$. Then $C_{s m}(I)$ denotes the set of all strictly monotone continuous functions on $I$.

Let $C_{\text {smof } f}(I)$ be the set of all $\phi \in C_{s m}(I)$ with $\phi \circ f \in L^{1}(\Omega, \mu)$. Let $\phi$ be an arbitrary function of $C_{\text {swof } f}(I)$. Since $\phi(I)$ is an interval of $\mathbb{R}$ and $\mu$ is a probability measure on $\Omega$, it follows that

$$
\int(\varphi \circ f) \mathrm{d} \mu \in \varphi(I) .
$$

Then there exists a unique real number $M_{\phi}(f) \in I$ such that $\int(\varphi \circ f) \mathrm{d} \mu=\varphi\left(M_{\varphi}(f)\right)$. Since $\phi$ is one-to-one, it follows that

$$
M_{\varphi}(f)=\varphi^{-1}\left(\int(\varphi \circ f) \mathrm{d} \mu\right) .
$$

(c) 2011 Nakasuji et al; licensee Springer. This is an Open Access article distributed under the terms of the Creative Commons Attribution License (http://creativecommons.org/licenses/by/2.0), which permits unrestricted use, distribution, and reproduction in any medium, provided the original work is properly cited. 
We call $M_{\phi}(f)$ a $\phi$-quasi-arithmetic mean of $f$ with respect to $\mu$ (or simply, $\phi$-mean of $f$ ). A $\phi$-mean of $f$ has the following invariant property:

$$
M_{\varphi}(f)=M_{a \varphi+b}(f)
$$

for each $a, b \in \mathbb{R}$ with $a \neq 0$.

Assume that $\mu\left(\Omega \backslash\left\{\omega_{1}, \ldots, \omega_{n}\right\}\right)=0$ for some $\omega_{1}, \ldots, \omega_{n} \in \Omega, f$ is a positive measurable function on $\Omega$ and $I=\mathbb{R}$. Then $M_{\phi}(f)$ will denote a weighted arithmetic mean, a weighted geometric mean, a weighted harmonic mean, etc. of $\left\{f\left(\omega_{1}\right), \ldots, f\left(\omega_{n}\right)\right\}$ if $\phi(x)=$ $x, \phi(x)=\log x, \varphi(x)=\frac{1}{x}$, etc., respectively.

In Section 2, we prepare some lemmas which we will need in the proof of our main results.

In Section 3, we first see that a $\phi$-mean function: $\nabla_{\phi} \rightarrow M_{\phi}(f)$ is order-preserving as a new interpretation of Jensen's inequality (see Theorem 1). We next see that there is a strictly monotone increasing $\phi$-mean (continuous) path between two $\phi$-means (see Theorem 2). We next see that the $\phi$-mean function is strictly concave (or convex) on a suitable convex subset of $C_{\text {smof }}(I)$ (see Theorem 3). We also observe a certain boundedness of $\phi$-means, more precisely,

$$
\sup _{s \geq 0} M_{(1-s) \varphi+s \psi}(f)=M_{\psi-\varphi}(f)
$$

under some conditions (see Theorem 4).

In Section 4, we treat a special $\phi$-mean in which $\phi$ is a $C^{2}$-functions with no stationary points.

In Section 5, we will give a new refinement of the geometric-arithmetic mean inequality as an application of our results.

\section{Lemmas}

This section is devoted to collecting some lemmas which we will need in the proof of our main results. The first lemma is to describe geometric properties of convex function, but this will be standard, so we will omit the proof (cf. [[2], (13.34) Exercise: Convex functions].

Lemma 1. Let $\phi$ be a real-valued function on I. Then the following three assertions are pairwise equivalent:

(i) $\phi$ is convex (resp. strictly convex).

(ii) For any $c \in I^{\circ}$, a function $\lambda_{c, \phi}$ defined by

$$
\lambda_{c, \varphi}(x)=\frac{\varphi(x)-\varphi(c)}{x-c} \quad(x \in I \backslash\{c\})
$$

is monotone increasing (resp. strictly monotone increasing) on $\Lambda\{c\}$.

(iii) For any $c \in I^{\circ}$, there is a real constant $m_{c} \in \mathbb{R}$ such that

$$
\varphi(x)-\varphi(c)-m_{c}(x-c) \geq 0 \quad(\text { resp. }>0)
$$

for all $x \in \Lambda\{c\}$, i.e., the line through $(c, \phi(c))$ having slope $m_{c}$ is always below or on (resp. below) the graph of $\phi$. 
Here $I^{\circ}$ denotes the interior of $I$.

For $\phi, \psi \in C_{s m}(I)$ and $c \in I^{\circ}$, put

$$
\lambda_{c, \varphi, \psi}(x)=\frac{\psi(x)-\psi(c)}{\varphi(x)-\varphi(c)} \quad(x \in I \backslash\{c\}) .
$$

This function has the following invariant property:

$$
\lambda_{c, \varphi, \psi}=\lambda_{c, a \varphi+b, a \psi+b}
$$

for each $a, b \in \mathbb{R}$ with $a \neq 0$. In this case, we have the following

Lemma 2. Let $\varphi, \psi \in C_{s m}^{+}(I)$. Then, the following three assertions are pairwise equivalent:

(i) For any $c \in I^{\circ}, \lambda_{c, \phi, \psi}$ is monotone increasing (resp. strictly monotone increasing) on $\Lambda\{c\}$.

(ii) For any $c \in I^{\circ}$, there is a real constant $m_{c} \in \mathbb{R}$ such that

$$
\psi(x)-\psi(c)-m_{c}(\varphi(x)-\varphi(c)) \geq 0 \quad(\text { resp. }>0)
$$

for all $x \in \Lambda\{c\}$.

(iii) $\psi \circ \phi^{-1}$ is convex (resp. strictly convex) on $\phi(I)$.

Proof. (i) $\Rightarrow$ (ii). Fix $c \in I^{\circ}$ arbitrarily. For any $x \in \Lambda\{c\}$, put $u=\phi(x)$ and then

$$
\left(\lambda_{c, \varphi, \psi} \circ \varphi^{-1}\right)(u)=\frac{\left(\psi \circ \varphi^{-1}\right)(u)-\left(\psi \circ \varphi^{-1}\right)(\varphi(c))}{u-\varphi(c)} .
$$

If $\lambda_{c, \phi, \psi}$ is monotone increasing (resp. strictly monotone increasing) on $I\{c\}$, then $\lambda_{c, \phi, \psi} \circ \phi^{-1}$ is also monotone increasing (resp. strictly monotone increasing) on $\phi(I)$ $\backslash\{\phi(c)\}$ and hence by (1) and Lemma 1 , we can find a real constant $m_{c} \in \mathbb{R}$ which is independent of $x$ such that

$$
\left(\psi \circ \varphi^{-1}\right)(u)-\left(\psi \circ \varphi^{-1}\right)(\varphi(c))-m_{c}(u-\varphi(c)) \geq 0 \quad(\text { resp. }>0) .
$$

Since $u=\phi(x)$, we have

$$
\psi(x)-\psi(c)-m_{c}(\varphi(x)-\varphi(c)) \geq 0 \quad(\text { resp. }>0) .
$$

(ii) $\Rightarrow$ (iii). Take $u \in \phi(I)$ and $d \in(\phi(I))^{\circ}$ arbitrarily. Put $x=\phi^{-1}(u)$ and $c=\phi^{-1}(d)$. Then $x \in I$ and $c \in I^{\circ}$. If we can find a real constant $m_{c} \in \mathbb{R}$ which is independent of $u$ such that

$$
\psi(x)-\psi(c)-m_{c}(\varphi(x)-\varphi(c)) \geq 0 \quad(\text { resp. }>0),
$$

then

$$
\left(\psi \circ \varphi^{-1}\right)(u)-\left(\psi \circ \varphi^{-1}\right)(d)-m_{c}(u-d) \geq 0 \quad(\text { resp. }>0),
$$

and hence $\psi \circ \phi^{-1}$ is convex (resp. strictly convex) on $\phi(I)$ by Lemma 1 .

(iii) $\Rightarrow$ (i). Take $c \in I^{\circ}$ and $x \in \Lambda\{$ c $\}$ arbitrarily. Put $u=\phi(x)$ and $d=\phi(c)$. 
Then $u \in \phi(I) \backslash\{d\}$ and $d \in(\phi(I))^{\circ}$, hence

$$
\left(\lambda_{c, \varphi, \psi} \circ \varphi^{-1}\right)(u)=\frac{\left(\psi \circ \varphi^{-1}\right)(u)-\left(\psi \circ \varphi^{-1}\right)(d)}{u-d} .
$$

If $\psi \circ \phi^{-1}$ is convex (resp. strictly convex) on $\phi(I)$, then by (2) and Lemma $11, \lambda_{c, \phi, \psi} \circ$ $\phi^{-1}$ and hence $\lambda_{c, \phi, \psi}$ is monotone increasing (resp. strictly monotone increasing) on $I$ $\backslash\{c\}$. ㅁ

For each $\phi \in C_{s m}(I), t \in[0,1]$ and $x, y \in I$, put

$$
x \nabla_{t, \varphi} y=\varphi^{-1}((1-t) \varphi(x)+t \varphi(\gamma)) .
$$

This can be regarded as a $\phi$-mean of $\{x, y\}$ with respect to a probability measure which represents a weighted arithmetic mean $(1-t) x+t y$.

For each $\phi \in C_{s m}(I)$, denote by $\nabla_{\phi}$ a three variable real-valued function:

$$
(t, x, y) \rightarrow x \nabla_{t, \varphi} y
$$

on $(0,1) \times\left\{(x, y) \in I^{2}: x \neq y\right\}$. For each $\phi, \psi \in C_{s m}(I)$, we write $\nabla_{\phi} \leq \nabla_{\psi}\left(\right.$ resp. $\left.\nabla_{\phi}<\nabla_{\psi}\right)$ if

$$
x \nabla_{t, \varphi} y \leq x \nabla_{t, \psi} \gamma \quad \text { (resp. } x \nabla_{t, \varphi} y<x \nabla_{t, \psi} y \text { ) }
$$

for all $t \in(0,1)$ and $x, y \in I$ with $x \neq y$.

Remark. The continuity of $\phi$ implies that $\nabla_{\phi} \leq \nabla_{\psi}$ (resp. $\nabla_{\phi}<\nabla_{\psi}$ ) if and only if

$$
x \nabla_{\frac{1}{2}, \varphi} y \leq x \nabla_{\frac{1}{2}, \psi} y \quad\left(\text { resp. } x \nabla_{\frac{1}{2}, \varphi} y<x \nabla_{\frac{1}{2}, \psi} y\right)
$$

for all $x, y \in I$ with $x \neq y$.

These order relations " $\leq$ " and " $<$ " play an important role in our discussion.

Lemma 3. Let $\phi, \psi L C_{s m}(I)$. Then

(i) $\nabla_{\phi}=\nabla_{\psi}$ holds if and only if $\psi=a \phi+b$ for some $a, b \in \mathbb{R}$ with $a \neq 0$.

(ii) If $\psi \in C_{s m}^{+}(I)$, then $\nabla_{\phi} \leq \nabla_{\psi}$ (resp. $\nabla_{\phi}<\nabla_{\psi}$ ) holds if and only if $\psi \circ \phi^{-1}$ is convex (resp. strictly convex) on $\phi(I)$.

(iii) If $\psi \in C_{s m}^{-}(I)$, then $\nabla_{\phi} \leq \nabla_{\psi}$ (resp. $\left.\nabla_{\phi}<\nabla_{\psi}\right)$ holds if and only if $\psi \circ \phi^{-1}$ is concave (resp. strictly concave) on $\phi(I)$.

Proof. (i) Suppose that $\nabla_{\phi}=\nabla_{\psi}$ holds. Take $u, v \in \phi(I)$ with $u \neq v$ arbitrarily and put $x=\phi^{-1}(u)$ and $y=\phi^{-1}(v)$, hence $x \neq y$. By hypothesis,

$$
\psi\left(\varphi^{-1}((1-t) u+t v)\right)=(1-t) \psi\left(\varphi^{-1}(u)\right)+t \psi\left(\varphi^{-1}(v)\right)
$$

for all $t \in(0,1)$. This means that $\psi^{\circ} \phi^{-1}$ is convex and concave on $\phi(I)$ and hence we can write $\psi\left(\phi^{-1}(u)\right)=a u+b$ for all $u \in \phi(I)$ and some $a, b \in \mathbb{R}$. Therefore, $\psi(x)=$ $a \phi(x)+b$ for all $x \in I$. Since $\psi$ is non-constant, it follows that $a \neq 0$.

The reverse assertion is straightforward.

(ii) Assume that $\psi$ is monotone increasing. Take $u, v \in \phi(I)$ with $u \neq v$ arbitrarily and put $x=\phi^{-1}(u)$ and $y=\phi^{-1}(v)$, hence $x \neq y$. If $\nabla_{\phi} \leq \nabla_{\psi}$ holds, then

$$
\psi\left(\varphi^{-1}((1-t) \varphi(x)+t \varphi(\gamma))\right) \leq(1-t) \psi(x)+t \psi(y)
$$


and hence

$$
\psi\left(\varphi^{-1}((1-t) u+t v)\right) \leq(1-t) \psi\left(\varphi^{-1}(u)\right)+t \psi\left(\varphi^{-1}(v)\right)
$$

for all $t \in(0,1)$. This means that $\psi^{\circ} \phi^{-1}$ is convex.

Conversely, if $\psi \circ \phi^{-1}$ is convex, we see that $\nabla_{\phi} \leq \nabla_{\psi}$ holds by observing the reverse of the above proof.

Also a similar observation implies that $\nabla_{\phi}<\nabla_{\psi}$ holds if and only if $\psi \circ \phi^{-1}$ is strictly convex on $I$.

(iii) Assume that $\psi$ is monotone decreasing. Then $-\psi$ is monotone increasing. Hence, by (ii), we have that $\nabla_{\phi} \leq \nabla_{-\psi}$ (resp. $\nabla_{\phi}<\nabla_{-\psi}$ ) holds if and only if $(-\psi) \circ \phi^{-1}$ is convex (resp. strictly convex) on $\phi(I)$. However, since $\nabla_{\psi}=\nabla_{-\psi}$ holds by (i) and $(-\psi) \circ \phi^{-1}$ is convex (resp. strictly convex) on $\phi(I)$ iff $\psi^{\circ} \phi^{-1}$ is concave (resp. strictly concave) on $\phi(I)$, we obtain the desired result.

Lemma 4. Let $\varphi, \psi \in C_{s m}^{+}(I)\left(\right.$ or $\left.C_{s m}^{-}(I)\right)$ with $\nabla_{\phi}<\nabla_{\psi}$. For each $\mathrm{s} \in[0,1]$, define $\xi_{s}=$ $(1-s) \phi+s \psi$. Then

(i) Each $\xi_{s}$ belongs to $C_{s m}^{+}(I)\left(\right.$ resp. $\left.C_{s m}^{-}(I)\right)$ when $\varphi, \psi \in C_{s m}^{+}(I)\left(\right.$ resp. $\left.C_{s m}^{-}(I)\right)$.

(ii) For each $t \in(0,1)$ and $x, y \in I$ with $x \neq y$, a function $s \rightarrow x \nabla_{t, \xi_{s}}$ yis strictly monotone increasing on $[0,1]$.

Proof. (i) Straightforward.

(ii) Assume $\varphi, \psi \in C_{s m}^{+}(I)$ with $\nabla_{\phi}<\nabla_{\psi}$. Take $t \in(0,1)$ and $x, y \in I$ with $x \neq y$ arbitrarily. To show that a function $s \rightarrow x \nabla_{t, \xi_{s}} y$ is strictly monotone increasing on $[0,1]$, let $0 \leq s_{1}<s_{2} \leq 1$. Take $c \in I^{\circ}$ arbitrarily. Since $\nabla_{\phi}<\nabla_{\psi}$ holds, it follows from Lemmas 2 and 3 that $\lambda_{c, \phi, \psi}$ is strictly monotone increasing on $\Lambda\{c\}$. Moreover, we have

$$
\begin{aligned}
\lambda_{c, \xi_{s_{1}}, \xi_{s_{2}}}(x) & =\frac{\xi_{s_{2}}(x)-\xi_{s_{2}}(c)}{\xi_{s_{1}}(x)-\xi_{s_{1}}(c)} \\
& =\frac{s_{2}(\psi(x)-\psi(c))+\left(1-s_{2}\right)(\varphi(x)-\varphi(c))}{s_{1}(\psi(x)-\psi(c))+\left(1-s_{1}\right)(\varphi(x)-\varphi(c))} \\
& =\frac{s_{2} \lambda_{c, \varphi, \psi}(x)+1-s_{2}}{s_{1} \lambda_{c, \varphi, \psi}(x)+1-s_{1}} .
\end{aligned}
$$

for each $\mathrm{x} \in \Lambda\{c\}$. Therefore, we have

$$
\lambda_{c, \xi_{s_{1}}, \xi_{s_{2}}}(x)=s_{2} \lambda_{c, \varphi, \psi}(x)+1-s_{2} \quad\left(s_{1}=0\right)
$$

and

$$
\lambda_{c, \xi_{s_{1}}, \xi_{s_{2}}}(x)=\frac{s_{2}}{s_{1}}-\frac{s_{2}-s_{1}}{s_{1}^{2}} \frac{1}{\lambda_{c, \varphi, \psi}(x)-\frac{s_{1}-1}{s_{1}}} \quad\left(s_{1} \neq 0\right)
$$

for each $\mathrm{x} \in \Lambda\{c\}$. If $s_{1}=0$, then it is trivial by (3) that $\lambda_{c, \xi_{s_{1}}, \xi_{s_{2}}}$ is strictly monotone increasing on $\Lambda\{c\}$. If $s_{1} \neq 0$, then

$$
\frac{s_{1}-1}{s_{1}}<0<\lambda_{c, \varphi \cdot \psi}(x)
$$

for all $x \in \Lambda\{c\}$. So, by (4), $\lambda_{c, \xi_{s_{1}}, \xi_{s_{2}}}$ is also strictly monotone increasing on $\Lambda\{c\}$. Hence we see that $\nabla_{\xi_{s_{1}}}<\nabla_{\xi_{s_{2}}}$ holds by (i), Lemmas 2 and 3 . This implies that 
$s \rightarrow x \nabla_{t, \xi_{s}} y$. Then a function $s \rightarrow x \nabla_{t, \xi_{s}} y$ is strictly monotone increasing on [0,1], as required.

For the case of $\varphi, \psi \in C_{s m}^{-}(I)$, since $-\varphi,-\psi \in C_{s m}^{+}(I)$, it follows from the above discussion that a function $s \rightarrow x \nabla_{t,-\xi_{s}} y$ is strictly monotone increasing on [0,1]. However, by Lemma 3-(i), $x \nabla_{t,-\xi_{s}} y=x \nabla_{t, \xi_{s}} y$, where $t \in(0,1)$ and $x, y \in I$ with $x \neq y$, and then we obtain the desired result. $\square$

Lemma 5. Let $\phi$ and $\psi$ be two functions on I such that $\psi-\phi$ is strictly monotone increasing (resp. decreasing) on I and $\psi$ is convex (resp. concave) on I. Then

$$
(1-t) \varphi(x)+t \psi(y)-((1-t) \varphi+t \psi)((1-t) x+t y)>0(\text { resp. }<0)
$$

holds for all $t \in(0,1)$ and $x, y \in I$ with $x<y$.

Proof. Let $x, y \in I$ with $x<y$ and $t \in(0,1)$. Put $z=(1-t) x+t y$. Then, we must show that $(1-t) \phi(x)+t \psi(y)-((1-t) \phi+t \psi)(z)>0$ (resp. $<0)$. Since $x<z<y$ and $\psi-\phi$ is strictly monotone increasing (resp. decreasing) on $I$, it follows that

$$
\psi(z)-\psi(x)-\varphi(z)+\varphi(x)>0(\text { resp. }<0) .
$$

Also since $\psi$ is convex (resp. concave) on $I$, it follows from Lemma 1 that $\lambda_{z, \psi}$ is monotone increasing (resp. decreasing). Therefore, we have

$$
\begin{aligned}
& (1-t) \varphi(x)+t \psi(y)-((1-t) \varphi+t \psi)(z) \\
& \quad=t(\psi(y)-\psi(z))-(1-t)(\varphi(z)-\varphi(x)) \\
& \quad>t(\psi(y)-\psi(z))-(1-t)(\psi(z)-\psi(x)) \\
& \quad(\text { resp. }<) \\
& \quad=t(1-t)(y-x)\left(\frac{\psi(y)-\psi(z)}{(1-t)(y-x)}-\frac{\psi(z)-\psi(x)}{t(y-x)}\right) \\
& \quad=t(1-t)(y-x)\left(\frac{\psi(y)-\psi(z)}{y-z}-\frac{\psi(x)-\psi(z)}{x-z}\right) \\
& \quad=t(1-t)(y-x)\left(\lambda_{z, \psi}(y)-\lambda_{z, \psi}(x)\right) \\
& \quad \geq 0 \\
& \quad(\text { resp. } \leq 0),
\end{aligned}
$$

so that $(1-t) \phi(x)+t \psi(y)-((1-t) \phi+t \psi)(z)>0$ (resp. $<0)$, as required.

The following lemma gives an equality condition of Jensen's inequality. For the sake of completeness, we will give a proof.

Lemma 6. Let $\delta$ be a strictly convex or strictly concave function on I. Suppose that $g$ is a real-valued integrable function on $\Omega$ such that $g(\omega) \in I$ for almost all $\omega \in \Omega$ and $\delta \circ g \in L^{1}(\Omega, \mu)$. Then $\delta\left(\int g d \mu\right)=\int(\delta \circ g) d \mu$ if and only if $g$ is a constant function.

Proof. We first consider the strictly convex case. Put $c=\int g \mathrm{~d} \mu$. If $c=\inf I$, then $c \leq g$ $(\omega)$ for almost all $\omega \in \Omega$ and so $g(\omega)=c$ must hold for almost all $\omega \in \Omega$. Similarly, if $c=\max I$, then $g(\omega)=c$ for almost all $\omega \in \Omega$. Therefore, we can without loss of generality assume that $c$ belongs to $I^{\circ}$. Since $\delta$ is strictly convex, we can from Lemma 1 find a real constant $m_{c} \in \mathbb{R}$ such that

$$
\delta(x)>m_{c}(x-c)+\delta(c)
$$

for all $x \in \Lambda\{c\}$. Replacing $x$ by $g(\omega)$ in (5), we obtain

$$
\delta(g(\omega)) \geq m_{c}(g(\omega)-c)+\delta(c)
$$


for almost all $\omega \in \Omega$. Integrating both sides of this equation, we have

$$
\int(\delta \circ g) \mathrm{d} \mu \geq \int\left(m_{c}(g-c)+\delta(c)\right) \mathrm{d} \mu=\delta(c)=\delta\left(\int g \mathrm{~d} \mu\right) .
$$

Now assume that $\delta\left(\int g \mathrm{~d} \mu\right)=\int(\delta \circ g) \mathrm{d} \mu$. Then (6) implies that

$$
\delta(g(\omega))=m_{c}(g(\omega)-c)+\delta(c)
$$

for almost all $\omega \in \Omega$. If $\mu(\{g \neq c\})>0$, then we can find $\omega_{c} \in \Omega$ such that $\delta\left(g\left(\omega_{c}\right)\right)=$ $m_{c}\left(g\left(\omega_{c}\right)-c\right)+\delta(c)$ and $g\left(\omega_{c}\right) \neq \mathrm{c}$. This contradicts (5) and hence $g(\omega)=c$ for almost all $\omega \in \Omega$.

Conversely, assume that $g$ is a constant function on $\Omega$. Then it is trivial that $\delta\left(\int g \mathrm{~d} \mu\right)=\int(\delta \circ g) \mathrm{d} \mu$.

For the strictly concave case, since $-\delta$ is strictly convex on $I$, it follows from the above discussion that $-\delta\left(\int g \mathrm{~d} \mu\right)=\int(-\delta \circ g) \mathrm{d} \mu$ iff $g$ is a constant function on $\Omega$. However, since $-\delta\left(\int g \mathrm{~d} \mu\right)=\int(-\delta \circ g) \mathrm{d} \mu$ iff $\delta\left(\int g \mathrm{~d} \mu\right)=\int(\delta \circ g) \mathrm{d} \mu$, we obtain the desired result. $\square$

Lemma 7. Suppose that $f$ is non-constant and $\phi, \psi \in C_{s m, f}(I)$. Then

(i) If either $\psi \circ \phi^{-1}$ is convex (resp. strictly convex) on $\phi(I)$ and $\psi \in C_{s m}^{+}(I)$ or $\psi \circ \phi^{-1}$ is concave (resp. strictly concave) on $\phi(I)$ and $\psi \in C_{s m}^{-}(I)$, then

$$
M_{\varphi}(f) \leq M_{\psi}(f) \quad\left(\operatorname{resp} . M_{\varphi}(f)<M_{\psi}(f)\right)
$$

holds.

(ii) If either $\psi \circ \phi^{-1}$ is convex (resp. strictly convex) on $\phi(I)$ and $\psi \in C_{s m}^{-}(I) o r \psi \circ \phi^{-1}$ is concave (resp. strictly concave) on $\phi(I)$ and $\psi \in C_{s m}^{+}(I)$, then

$$
M_{\varphi}(f) \geq M_{\psi}(f) \quad\left(\operatorname{resp} . M_{\varphi}(f)>M_{\psi}(f)\right)
$$

holds.

Proof. (i) Put $\delta=\psi \circ \phi^{-1}$ and $g=\phi \circ f$. Assume that $g$ is convex on $\phi(I)$ and $\psi \in C_{s m}^{+}(I)$. Since $g$ and $\delta \circ g$ are integrable functions on $\Omega$, we have

$$
\delta\left(\int g \mathrm{~d} \mu\right) \leq \int(\delta \circ g) \mathrm{d} \mu
$$

by Jensen's inequality. This means $M_{\phi}(f) \leq M_{\psi}(f)$ because $\psi$ is monotone increasing on $I$.

Next assume that $g$ is concave on $\phi(I)$ and $\psi \in C_{s m}^{-}(I)$. Then

$$
\delta\left(\int g \mathrm{~d} \mu\right) \geq \int(\delta \circ g) \mathrm{d} \mu
$$

by Jensen's inequality. This also means $M_{\phi}(f) \leq M_{\psi}(f)$ because $\psi$ is monotone decreasing on $I$.

For the strict case, since $g$ is a non-constant function on $\Omega$, we obtain the desired results from (7), (8), Lemma 6 and the above argument. $\square$

(ii) Similarly. 


\section{Main results}

In this section, we first give a new interpretation of Jensen's inequality by $\phi$-mean. Next, as an application, we consider some geometric properties of $\phi$-means of a realvalued measurable function $f$ on $\Omega$.

The first result asserts that a $\phi$-mean function: $\nabla_{\phi} \rightarrow M_{\phi}(f)$ is well defined and order preserving, and this assertion simultaneously gives a new interpretation of Jensen's inequality. However, this assertion also teaches us that a simple inequality yields a complicated inequality.

Theorem 1. Suppose that $f$ is non-constant and $\phi, \psi \in C_{s m, f}(I)$. Then

(i) If $\nabla_{\phi} \leq \nabla_{\psi}$ holds, then $M_{\phi}(f) \leq M_{\psi}(f)$.

(ii) If $\nabla_{\phi}<\nabla_{\psi}$ holds, then $M_{\phi}(f)<M_{\psi}(f)$.

Proof. (i) Suppose that $\nabla_{\phi} \leq \nabla_{\psi}$ holds. If $\psi$ is monotone increasing on $I$, then $\psi \circ \phi^{-1}$ is convex on $\phi(I)$ by Lemma 3-(ii). Therefore, we have $M_{\phi}(f) \leq M_{\psi}(f)$ by Lemma 7-(i). If $\psi$ is monotone decreasing on $I$, then $\psi \circ \phi^{-1}$ is concave on $\phi(I)$ by Lemma 3-(iii). Therefore, we have $M_{\phi}(f) \leq M_{\psi}(f)$ by Lemma 7-(i).

(ii) Similarly.

Let $\phi, \psi \in C_{s m, f}(I)$ and $t \in(0,1)$. Then, we can easily see that if either both $\phi$ and $\psi$ are monotone increasing or both $\phi$ and $\psi$ are monotone decreasing, then $(1-t) \phi+t \psi$ is also an element of $C_{s m, f}(I)$ [cf. Lemma 4-(i)]. The next result asserts that there is a strictly monotone increasing $\phi$-mean (continuous) path between two $\phi$-means.

Theorem 2. Suppose that $f$ is non-constant and $\phi, \psi \in C_{s m, f}(I)$ with $\nabla_{\phi}<\nabla_{\psi}$.

(i) If $\varphi, \psi \in C_{s m}^{+}(I)\left[\right.$ or $\left.C_{s m}^{-}(I)\right]$, then a function: $\mathrm{s} \rightarrow M_{(1-s) \phi+s \psi}(f)$ is strictly monotone increasing on $[0,1]$.

(ii) If $\varphi, \psi-\varphi \in C_{s m}^{+}(I)$ [resp. $\left.C_{s m}^{-}(I)\right]$ and $\psi(x)-\phi(x) \geq 0$ (resp. $\left.\leq 0\right)$ for all $x \in I$, then a function: $\mathrm{s} \rightarrow M_{(1-s) \phi+s \psi}(f)$ is strictly monotone increasing and continuous on $[0.1]$.

Proof. (i) Suppose that $\varphi, \psi \in C_{s m}^{+}(I)\left[\right.$ or $\left.C_{s m}^{-}(I)\right]$. For each $s \in[0,1]$, define $\xi_{s}=(1-s) \phi+$ $s_{\psi}$. Let $0 \leq s_{1}<s_{2} \leq 1$. Then, we must show that $M_{\xi_{s_{1}}}(f)<M_{\xi_{s_{2}}}(f)$. By Lemma 4-(ii), a function $s \rightarrow x \nabla_{t, \xi_{s}} y$ is strictly monotone increasing on [0,1] for each $t \in(0,1)$ and $x, y \in$ $I$ with $x \neq y$, and hence we see that $\nabla_{\xi_{s_{1}}}<\nabla_{\xi_{s_{2}}}$ holds. Therefore, we have from Theorem 1-(ii) that $M_{\xi_{s_{1}}}(f)<M_{\xi_{s_{2}}}(f)$, as required.

(ii) Suppose that $\varphi, \psi-\varphi \in C_{s m}^{+}(I)$ and $\phi(x) \leq \psi(x)$ for all $x \in I$. Since $\psi=\phi+(\psi-\phi)$, it follows that $\psi \in C_{s m}^{+}(I)$. For each $s \in[0,1]$, put $\alpha_{s}=\mathrm{M}_{(1-s) \phi+s \psi}(f)$. Then, we must show that a function $s \rightarrow \alpha_{s}$ is continuous on [0,1]. To do this, take $0 \leq s<t \leq 1$ arbitrarily. By (i), we have $\alpha_{s}<\alpha_{t}$. Note that

$$
(1-t) \varphi\left(\alpha_{t}\right)+t \psi\left(\alpha_{t}\right)=(1-t) \int(\varphi \circ f) \mathrm{d} \mu+t \int(\psi \circ f) \mathrm{d} \mu
$$

and

$$
(1-s) \varphi\left(\alpha_{s}\right)+s \psi\left(\alpha_{s}\right)=(1-s) \int(\varphi \circ f) \mathrm{d} \mu+s \int(\psi \circ f) \mathrm{d} \mu .
$$


Therefore, we have

$$
\varphi\left(\alpha_{t}\right)-\varphi\left(\alpha_{s}\right)+t(\psi-\varphi)\left(\alpha_{t}\right)-s(\psi-\varphi)\left(\alpha_{s}\right)=(t-s)\left(\int((\psi-\varphi) \circ f) \mathrm{d} \mu\right) .
$$

Since $\varphi, \psi-\varphi \in C_{s m}^{+}(I)$ and $\phi(x) \leq \psi(x)$ for all $x \in I$ by hypothesis, it follows that

$$
\varphi\left(\alpha_{t}\right)-\varphi\left(\alpha_{s}\right)>0 \text { and } t(\psi-\varphi)\left(\alpha_{t}\right)-s(\psi-\varphi)\left(\alpha_{s}\right)>0 \text {. }
$$

Hence, after taking the limit with respect to $s$ in the Eq. (9), we obtain

$$
\lim _{s \rightarrow t-0} \varphi\left(\alpha_{s}\right)=\varphi\left(\alpha_{t}\right) \text { and } \lim _{s \rightarrow t-0} s(\psi-\varphi)\left(\alpha_{s}\right)=t(\psi-\varphi)\left(\alpha_{t}\right) .
$$

However, since $\phi^{-1}$ is continuous on $\phi(I)$, we conclude that

$$
\lim _{s \rightarrow t-0} \alpha_{s}=\alpha_{t}
$$

Similarly, after taking the limit with respect to $t$ in the Eq. (9), we obtain

$$
\lim _{t \rightarrow s+0} \alpha_{t}=\alpha_{s}
$$

These observations imply that a function $s \rightarrow \alpha_{s}$ is continuous on $[0,1]$, as required.

For the case that $\varphi, \psi-\varphi \in C_{s m}^{-}(I)$ and $\phi(x) \geq \psi(x)$ for all $x \in I$, a similar argument above implies that a function $s \rightarrow \alpha_{s}$ is also continuous on $[0,1]$.

The next result asserts that the $\phi$-mean function is strictly concave (or convex) on a suitable convex subset of $C_{s m, f}(I)$.

Theorem 3. Suppose that $f$ is non-constant and $\phi, \psi \in C_{s m, f}(I)$ with $\nabla_{\phi}<\nabla_{\psi}$. Then

(i) If $\varphi, \psi-\varphi \in C_{s m}^{+}(I)\left(\right.$ resp. $\left.C_{s m}^{-}(I)\right)$ and $\psi$ is convex (resp. concave) on $I$, then

$$
(1-t) M_{\varphi}(f)+t M_{\psi}(f)<M_{(1-t) \varphi+t \psi}(f)
$$

holds for all $t \in(0,1)$.

(ii) If $\psi, \varphi-\psi \in C_{s m}^{-}(I)\left(r e s p . C_{s m}^{+}(I)\right)$ and $\psi$ is convex (resp. concave) on $I$, then

$$
(1-t) M_{\varphi}(f)+t M_{\psi}(f)>M_{(1-t) \varphi+t \psi}(f)
$$

holds for all $t \in(0,1)$.

Proof. (i) Suppose that $\varphi, \psi-\varphi \in C_{s m}^{+}(I)\left[\right.$ resp. $\left.C_{s m}^{-}(I)\right]$ and $\psi$ is convex [resp. concave] on $I$. Since $\psi=\phi+(\psi-\phi)$, it follows from hypothesis that $\psi \in C_{s m}^{+}(I)$ [resp. $\left.C_{s m}^{-}(I)\right]$. Put $x=M_{\phi}(f)$ and $y=M_{\psi}(f)$, and so $x<y$ by Theorem 1 -(ii). Also, we have from definition that

$$
\varphi(x)=\int(\varphi \circ f) \mathrm{d} \mu \text { and } \psi(\gamma)=\int(\psi \circ f) \mathrm{d} \mu .
$$

Let $0<t<1$ and put $u=M_{(1-t) \phi+t \psi}(f)$. Then, we have

$$
((1-t) \varphi+t \psi)(u)=\int(((1-t) \varphi+t \psi) \circ f) \mathrm{d} \mu
$$

by definition. Therefore,

$$
\begin{aligned}
(1-t) \varphi(x)+t \psi(\gamma) & =(1-t) \int(\varphi \circ f) \mathrm{d} \mu+t \int(\psi \circ f) \mathrm{d} \mu \\
& =\int(((1-t) \varphi+t \psi) \circ f) \mathrm{d} \mu \\
& =((1-t) \varphi+t \psi)(u) .
\end{aligned}
$$


Put $z=(1-t) x+t y$. Then, by the above equality and Lemma 5 , we have

$$
((1-t) \varphi+t \psi)(z)<(\text { resp. }>)(1-t) \varphi(x)+t \psi(\gamma)=((1-t) \varphi+t \psi)(u) .
$$

Since $(1-t) \phi+t \psi$ is strictly increasing (resp. decreasing), it follows that $z<u$, that is,

$$
(1-t) x+t y<u
$$

This means that $(1-t) M_{\phi}(f)+t M_{\psi}(f)<M_{(1-t) \phi+t \psi}(f)$.

(ii) Similarly.

Remark. It seems that Theorem 3 is slightly related to [3,4] which discuss a comparison between a convex linear combination of the arithmetic and geometric means and the generalized logarithmic mean.

The following result describes a certain boundedness of $\phi$-means.

Theorem 4. Suppose that $f$ is non-constant and $\phi, \psi \in C_{s m, f}(I)$ with $\nabla_{\phi}<\nabla_{\psi}$.

(i) If $\varphi, \psi-\varphi \in C_{s m}^{+}(I)$ [or $\left.C_{s m}^{-}(I)\right]$, then a function: $s \rightarrow M_{(1-s) \phi+s \psi}(f)$ is strictly monotone increasing on $[0, \infty)$ and

$$
\lim _{s \rightarrow \infty} M_{(1-s) \varphi+s \psi}(f)=M_{\psi-\varphi}(f) .
$$

(ii) If $\varphi, \psi-\varphi \in C_{s m}^{+}(I)\left[\right.$ resp. $\left.C_{s m}^{-}(I)\right]$ and $\psi(x)-\phi(x) \geq 0$ (resp. $\left.\leq 0\right)$ for all $x \in I$, then a function: $s \rightarrow M_{(1-s) \phi+s \psi}(f)$ is strictly monotone increasing and continuous on $[0, \infty)$.

Proof. (i) Suppose that $\varphi, \psi-\varphi \in C_{s m}^{+}(I)$. For each $s \geq 1$, put $\xi_{s}=(1-s) \phi+s \psi$. Since $\xi_{s}=\phi+s(\psi-\phi)$, it follows from hypothesis that each $\xi_{s}$ is in $C_{s m}^{+}(I)$, and then $\xi_{s} \in C_{s m}$, ${ }_{f}(I)$. Since $\psi=\phi+(\psi-\phi)$, it follows from hypothesis that $\psi$ is also in $C_{s m}^{+}(I)$. Then by Lemmas 2 and 3, we have that $\lambda_{c, \phi, \psi}$ is strictly monotone increasing on $I\{c\}$ for any $c$ $\in I^{\circ}$. Let $1 \leq s_{1}<s_{2}<\infty$ and take $c \in I^{\circ}$ arbitrarily. In this case, we obtain the equality (4), as observe in the proof of Lemma 4-(ii). Note that

$$
\frac{s_{1}-1}{s_{1}}<1<\lambda_{c, \varphi, \psi}(x)
$$

for all $x \in \Lambda\{c\}$. So, by (4), $\lambda_{c, \xi_{\xi_{1}}, \xi_{s_{2}}}$ is also strictly monotone increasing on $\Lambda\{c\}$. Then by Lemmas 2 and 3, we conclude that $\nabla_{\xi_{s_{1}}}<\nabla_{\xi_{s_{2}}}$. Therefore, we have from Theorem 1-(ii) that $M_{\xi_{s_{1}}}(f)<M_{\xi_{s_{2}}}(f)$ and then a function: $s \rightarrow M_{(1-s) \phi+s \psi}(f)$ is strictly monotone increasing on $[1, \infty)$ and hence $[0, \infty)$ by Theorem 2 -(i).

Moreover, we can easily see that

$$
\lambda_{c, \xi_{s}, \psi-\varphi}(x)=\frac{1}{s}-\frac{1}{s^{2}} \frac{1}{\lambda_{c, \varphi, \psi}(x)-\frac{s-1}{s}}
$$

and

$$
\frac{s-1}{s}<1<\lambda_{c, \varphi, \psi}(x)
$$


for all $s \geq 1, x \in I\{c\}$ and $c \in I^{\circ}$. This implies that $\lambda_{c, \xi_{s}, \psi-\varphi}$ is strictly monotone increasing on $\Lambda\{c\}$ for each $s \geq 1$ and $c \in I^{\circ}$. Then by Lemmas 2 and 3, we conclude that $\nabla_{\xi_{s}}<\nabla_{\psi-\varphi}$ for each $s \geq 1$. Therefore, we have from Theorem 1-(ii) that $M_{\xi_{s}}(f)<M_{\psi-\varphi}(f)$ for each $s \geq 1$.

Now take $s \geq 1$ arbitrarily and put $\alpha_{s}=M_{\xi_{s}}(f)$ and $\alpha=M_{\psi-\phi}(f)$, so $\alpha_{s}<\alpha$. Since $\varphi, \psi-\varphi \in C_{s m}^{+}(I)$, it follows that $\phi\left(\alpha_{s}\right)<\phi(\alpha)$ and $(\psi-\phi)\left(\alpha_{s}\right)<(\psi-\phi)(\alpha)$. By definition, we have

$$
(\psi-\varphi)(\alpha)=\int((\psi-\varphi) \circ f) \mathrm{d} \mu .
$$

Also since $\xi_{s}=s\left(\frac{1}{s} \varphi+\psi-\varphi\right)$, it follows from an invariant property of $\phi$-mean that $\alpha_{s}=M_{\frac{1}{s} \varphi+\psi-\varphi}(f)$ and then

$$
\frac{1}{s} \varphi\left(\alpha_{s}\right)+(\psi-\varphi)\left(\alpha_{s}\right)=\int\left(\frac{1}{s} \varphi+\psi-\varphi\right) \circ f \mathrm{~d} \mu
$$

Therefore, we have

$$
\begin{aligned}
0 & <(\psi-\varphi)(\alpha)-(\psi-\varphi)\left(\alpha_{s}\right) \\
& =\frac{1}{s}\left(\varphi\left(\alpha_{s}\right)-\int(\varphi \circ f) \mathrm{d} \mu\right) \\
& <\frac{1}{s}\left(\varphi(\alpha)-\int(\varphi \circ f) \mathrm{d} \mu\right) .
\end{aligned}
$$

Hence, after taking the limit with respect to $s$, we obtain

$$
\lim _{s \rightarrow \infty}(\psi-\varphi)\left(\alpha_{s}\right)=(\psi-\varphi)(\alpha) .
$$

However, since $(\psi-\phi)^{-1}$ is continuous on $(\psi-\phi)(I)$, we conclude that

$$
\lim _{s \rightarrow \infty} \alpha_{s}=\alpha,
$$

that is,

$$
\lim _{s \rightarrow \infty} M_{(1-s) \varphi+s \psi}(f)=M_{\psi-\varphi}(f) .
$$

For the decreasing case, replacing $\phi$ and $\psi$ by $-\phi$ and $-\psi$, respectively, apply the above discussion for the increasing case.

(ii) Refer to the Proof of Theorem 2-(ii). $\square$

\section{4. $\phi$-means by $\boldsymbol{C}^{2}$-functions}

In this section, we treat a special $\phi$-mean in which $\phi$ is a $C^{2}$-functions with no stationary points. For each real-valued measurable function $f$ on $\Omega$, let $C_{s m * f}^{2}(I)$ be the set of all $C^{2}$-functions $\phi$ in $C_{s m, f}(I)$ with no stationary points, that is, $\phi^{\prime}(t) \neq 0$ for all $t \in I$.

Lemma 8. Let $\varphi, \psi \in C_{s m *, f}^{2}(I)$. Then

(i) The following two statements are equivalent:
(a) $\psi \circ \phi^{-1}$ is convex (resp. concave) on $\phi(I)$.
(b) $\left(\frac{\psi^{\prime \prime}(x)}{\psi^{\prime}(x)}-\frac{\varphi^{\prime \prime}(x)}{\varphi^{\prime}(x)}\right) \psi^{\prime}(x) \geq 0$ (resp. $\left.\leq 0\right)$ for all $x \in I^{\circ}$.

(ii) The following two statements are equivalent: 
(c) $\psi \circ \phi^{-1}$ is strictly convex (resp. strictly concave) on $\phi(I)$.

(d) $\left(\frac{\psi^{\prime \prime}(x)}{\psi^{\prime}(x)}-\frac{\varphi^{\prime \prime}(x)}{\varphi^{\prime}(x)}\right) \psi^{\prime}(x)>0($ resp. $<0)$ for all $x \in I^{\circ}$.

Proof. (i) Define $\tau(u)=\psi\left(\left(\phi^{-1}(u)\right)\right.$ for each $u \in \phi(I)$. Then a simple calculation yields that

$$
\tau^{\prime \prime}(u)=\frac{\left(\frac{\psi^{\prime \prime}(x)}{\psi^{\prime}(x)}-\frac{\varphi^{\prime \prime}(x)}{\varphi^{\prime}(x)}\right) \psi^{\prime}(x)}{\left(\varphi^{\prime}(x)\right)^{2}}
$$

for all $u \in(\phi(I))^{\circ}$, where $x=\phi^{-1}(u)$. This equation implies that (a) and (b) are equivalent.

(ii) Similarly. $\square$

Lemma 9. Let $\phi$ and $\psi$ be $C^{1}$-functions on I. Then,

(i) If $\phi^{\prime}(x)<\psi^{\prime}(x)$ for all $x \in I^{\circ}$ and $\psi^{\prime}$ is monotone increasing on I, then

$$
((1-t) \varphi+t \psi)((1-t) x+t y)<(1-t) \varphi(x)+t \psi(y)
$$

holds for all $x, y \in I$ with $x<y$ and $t \in(0,1)$.

(ii) If $\phi^{\prime}(x)>\psi^{\prime}(x)$ for all $x \in I^{\circ}$ and $\psi^{\prime}$ is monotone decreasing on I, then

$$
((1-t) \varphi+t \psi)((1-t) x+t y)>(1-t) \varphi(x)+t \psi(y)
$$

holds for all $x, y \in I$ with $x<y$ and $t \in(0,1)$.

Proof. (i) Suppose that $\phi^{\prime}(x)<\psi^{\prime}(x)$ for all $x \in I^{\circ}$ and $\psi^{\prime}$ is monotone increasing on $I$. Let $x, y \in I$ with $x<y$ and $t \in(0,1)$. Put $z=(1-t) x+t y$. Then, we must show that $((1-t) \phi+t \psi)(z)<(1-t) \phi(x)+t \psi(y)$. By the mean value theorem, we have

$$
\begin{aligned}
& (1-t) \varphi(x)+t \psi(\gamma)-((1-t) \varphi+t \psi)(z) \\
& \quad=t(\psi(\gamma)-\psi(z))-(1-t)(\varphi(z)-\varphi(x)) \\
& \quad=t \psi^{\prime}(z+(y-z) \theta)(y-z)-(1-t) \varphi^{\prime}\left(x+(z-x) \theta^{\prime}\right)(z-x) \\
& \quad=t(1-t)\left(\psi^{\prime}(z+(y-z) \theta)-\varphi^{\prime}\left(x+(z-x) \theta^{\prime}\right)\right)(y-x) \\
& \quad \geq t(1-t)\left(\psi^{\prime}\left(x+(z-x) \theta^{\prime}\right)-\varphi^{\prime}\left(x+(z-x) \theta^{\prime}\right)\right)(y-x)
\end{aligned}
$$

for some $\theta, \theta^{\prime} \in(0,1)$ because $z+(y-z) \theta \geq x+(z-x) \theta^{\prime}$ and hence $\psi^{\prime}(z+(y-z) \theta) \geq \psi^{\prime}($ $\left.x+(z-x) \theta^{\prime}\right)$ by hypothesis. Since $x+(z-x) \theta^{\prime} \in I^{\circ}$, it follows from hypothesis that

$$
\psi^{\prime}\left(x+(z-x) \theta^{\prime}\right)>\varphi^{\prime}\left(x+(z-x) \theta^{\prime}\right)
$$

and so $(1-t) \phi(x)+t \psi(y)-((1-t) \phi+t \psi)(z)>0$ from the preceding inequalities. Therefore, we obtain the desired inequality.

(ii) Similarly. $\square$ 
Corollary 1. Suppose that $f$ is non-constant and $\varphi, \psi \in C_{s m *, f}^{2}(I)$. Then

(i) If $\frac{\varphi^{\prime \prime}(x)}{\varphi^{\prime}(x)} \leq \frac{\psi^{\prime \prime}(x)}{\psi^{\prime}(x)}$ for all $x \in I^{\circ}$, then $M_{\phi}(f) \leq M_{\psi}(f)$.

(ii) If $\frac{\varphi^{\prime \prime}(x)}{\varphi^{\prime}(x)}<\frac{\psi^{\prime \prime}(x)}{\psi^{\prime}(x)}$ for all $x \in I^{\circ}$ then $M_{\phi}(f)<M_{\psi}(f)$.

Proof. (i) Suppose that $\frac{\varphi^{\prime \prime}(x)}{\varphi^{\prime}(x)} \leq \frac{\psi^{\prime \prime}(x)}{\psi^{\prime}(x)}$ for all $x \in I^{\circ}$. If $\psi$ is monotone increasing on $I$, then $\psi^{\prime}(x)>0$ for all $x \in I^{\circ}$ and hence $\psi^{\circ} \phi^{-1}$ is convex on $\phi(I)$ by Lemma 8-(i). Therefore, by Lemma 3-(ii), $\nabla_{\phi} \leq \nabla_{\psi}$ holds and then $M_{\phi}(f) \leq M_{\psi}(f)$ by Theorem 1-(i). If $\psi$ is monotone decreasing on $I$, then $\psi^{\prime}(x)<0$ for all $x \in I^{\circ}$ and hence $\psi^{\circ} \phi^{-1}$ is concave on $\phi(I)$ by Lemma 8-(i). Therefore, by Lemma 3-(iii), $\nabla_{\phi} \leq \nabla_{\psi}$ also holds and then $M_{\phi}(f) \leq M_{\psi}(f)$ by Theorem 1-(i).

(ii) Similarly. $\square$

$\operatorname{Remark}$. Let $(\Omega, \mu)$ be a probability space, $0<p<q<\infty$ and let $f$ be a non-constant real-valued function in $L^{q}(\Omega, \mu)$. Then the well-known inequality: $\|f\|_{p}<\|f\|_{q}$ follows immediately from Corollary 1 (ii), by considering a family $\left\{\phi_{r}: r>0\right\}$ of functions on $\mathbb{R}_{+}$, where $\phi_{r}(x)=x^{r}(x>0)$.

Let $\varphi, \psi \in C_{s m *, f}^{2}(I)$ and let $t \in(0,1)$. Then, we can easily see that if either both $\phi$ and $\psi$ are monotone increasing on $I$ or both $\phi$ and $\psi$ are monotone decreasing on $I$, then $(1-t) \varphi+t \psi \in C_{s m *, f}^{2}(I)$. In this case, we have the following

Corollary 2. Suppose that $f$ is non-constant and $\varphi, \psi \in C_{s m *, f}^{2}(I)$. If $\frac{\varphi^{\prime \prime}(x)}{\varphi^{\prime}(x)}<\frac{\psi^{\prime \prime}(x)}{\psi^{\prime}(x)}$ and $\phi^{\prime}(x) \psi^{\prime}(x)>0$ for all $x \in I^{\circ}$, then a function: $s \rightarrow M_{(1-s) \phi+s \psi}(f)$ is strictly increasing on $[0,1]$.

Proof. Suppose that $\frac{\varphi^{\prime \prime}(x)}{\varphi^{\prime}(x)}<\frac{\psi^{\prime \prime}(x)}{\psi^{\prime}(x)}$ and $\phi^{\prime}(x) \psi^{\prime}(x)>0$ for all $x \in I^{\circ}$. We define $\xi(s, x)=$ $(1-s) \phi(x)+s \psi(x)$ for each $s \in(0,1)$. We can easily see that

$$
\frac{\frac{\partial^{2}}{\partial x^{2}} \xi(s, x)}{\frac{\partial}{\partial x} \xi(s, x)}-\frac{\varphi^{\prime \prime}(x)}{\varphi^{\prime}(x)}=\frac{s \psi^{\prime}(x)\left(\frac{\psi^{\prime \prime}(x)}{\psi^{\prime}(x)}-\frac{\varphi^{\prime \prime}(x)}{\varphi^{\prime}(x)}\right)}{(1-s) \varphi^{\prime}(x)+s \psi^{\prime}(x)}>0
$$

for each $s \in(0,1)$ and $x \in I^{\circ}$. Then, we have from Corollary 1-(ii) that $M_{\phi}(f)<M_{(1-s) \phi}$ $+s \psi(f)$ for all $s \in(0,1)$. Similarly, we can see that $M_{(1-s) \phi+s \psi}(f)<M_{\psi}(f)$ for all $s \in(0,1)$. Now put

$$
A(s, x)=\frac{\frac{\partial^{2}}{\partial x^{2}} \xi(s, x)}{\frac{\partial}{\partial x} \xi(s, x)}
$$

for each $s \in(0,1)$ and $x \in I^{\circ}$. Then a simple calculation implies that

$$
\frac{\partial}{\partial s} A(s, x)=\frac{\left(\frac{\psi^{\prime \prime}(x)}{\psi^{\prime}(x)}-\frac{\varphi^{\prime \prime}(x)}{\varphi^{\prime}(x)}\right) \varphi^{\prime}(x) \psi^{\prime}(x)}{\left((1-t) \varphi^{\prime}(x)+t \psi^{\prime}(x)\right)^{2}}>0
$$

for each $s \in(0,1)$ and $x \in I^{\circ}$. Therefore, for a fixed $x \in I^{\circ}$, a function: $s \rightarrow A(s, x)$ is strictly increasing on $(0,1)$. Therefore, Corollary 1-(ii) implies that a function: $s \rightarrow M$ $(1-s) \phi+s \psi(f)$ is strictly increasing on $(0,1)$ and hence $[0,1]$. 
Corollary 3. Suppose that $f$ is non-constant and that $\varphi, \psi \in C_{s m *, f}^{2}(I)$ is such that $\frac{\varphi^{\prime \prime}(x)}{\varphi^{\prime}(x)}<\frac{\psi^{\prime \prime}(x)}{\psi^{\prime}(x)}$ for for all $x \in I^{\circ}$. Then

(i) If either $0<\phi^{\prime}<\psi^{\prime}$ and $\psi^{\prime \prime} \geq 0$ on $I^{\circ}$ or $\psi^{\prime}<\phi^{\prime}<0$ and $\psi^{\prime \prime} \leq 0$ on $I^{\circ}$, then

$$
(1-t) M_{\varphi}(f)+t M_{\psi}(f)<M_{(1-t) \varphi+t \psi}(f)
$$

holds for all $t \in(0,1)$.

(ii) If either $\phi^{\prime}<\psi^{\prime}<0$ and $\psi^{\prime \prime} \geq 0$ on $I^{\circ}$ or $0<\psi^{\prime}<\phi^{\prime}$ and $\psi^{\prime \prime} \leq 0$ on $I^{\circ}$, then

$$
(1-t) M_{\varphi}(f)+t M_{\psi}(f)>M_{(1-t) \varphi+t \psi}(f)
$$

holds for all $t \in(0,1)$.

Proof. (i) Suppose that $0<\phi^{\prime}<\psi^{\prime}$ and $\psi^{\prime \prime} \geq 0$ on $I^{\circ}$. Put $x=M_{\phi}(f)$ and $y=M_{\psi}(f)$, and so $x<y$ by Corollary 1-(ii). Take $t \in \mathbb{R}$ with $0<t<1$ arbitrarily. By hypothesis, $(1-t) \phi$ $+t \psi$ is strictly monotone increasing on $I$. Put $u=M_{(1-t) \phi+t \psi}(f)$. As observe in the proof of Theorem 3-(i), we have

$$
(1-t) \varphi(x)+t \psi(\gamma)=((1-t) \varphi+t \psi)(u) .
$$

Put $z=(1-t) x+t y$. Then, by (10) and Lemma 9-(i), we have

$$
((1-t) \varphi+t \psi)(z)<(1-t) \varphi(x)+t \psi(\gamma)=((1-t) \varphi+t \psi)(u),
$$

and then $z<u$, that is, $(1-t) M_{\phi}(f)+t M_{\psi}(f)<M_{(1-t) \phi+t \psi}(f)$.

In the case of $\psi^{\prime}<\phi^{\prime}<0$ and $\psi \leq 0$ on $I^{\circ}$, we apply Lemma 9 -(ii).

(2) Similarly. $\square$

\section{Remarks}

(i) Let $I=\mathbb{R}^{+}$. Put $\varphi(x)=\frac{1}{x}$ and $\psi(x)=x$ for each $x \in I$. Of course, these functions belong to $C_{s m}(I)$. The harmonic-arithmetic mean inequality asserts that $\nabla_{\phi}<\nabla_{\psi}$. Take a non-constant positive measurable function $f$ on a probability space $(\Omega, \mu)$ such that $\phi \circ f$ and $\psi \circ f$ are in $L^{1}(\Omega, \mu)$. Then, we have from Theorem 1-(ii) that $M_{\phi}(f)<M_{\psi}(f)$. Observe that this inequality means

$$
1<\left(\int \frac{1}{f} \mathrm{~d} \mu\right)\left(\int f \mathrm{~d} \mu\right) .
$$

This is a special case of Jensen's inequality (or Schwarz's inequality). We note that if $0<m \leq f \leq M$, then $\left(\int \frac{1}{f} \mathrm{~d} \mu\right)\left(\int f \mathrm{~d} \mu\right) \leq \frac{(m+M)^{2}}{4 m M}$. The right side of this inequality is called a Kantorovich constant (cf. [5-7]).

(ii) A similar consideration for the geometric-arithmetic mean inequality yields that

$$
\int \log f \mathrm{~d} \mu<\log \int f \mathrm{~d} \mu .
$$


This is also a special case of Jensen's inequality. We note that if $0<m \leq f \leq M$, then $\log \int f \mathrm{~d} \mu-\int \log f \mathrm{~d} \mu \leq h^{\frac{1}{h-1}}\left(e \log h^{\frac{1}{h-1}}\right)^{-1}$, where $h=\frac{M}{m}$. The right side of this inequality is called Specht's ratio (cf. [8]).

(iii) For each $t \in[0,1]$, put $\log _{[t]} x=(1-t) \log x+t x(x>0)$. Then $\log _{[t]}$ is a strictly monotone increasing real-valued continuous function on $\mathbb{R}_{+}$. Denote by $\exp _{[t]}$ the inverse function of $\log _{[t]}$. Let $x_{1}, \ldots, x_{n}>0$ and $p_{1}, \ldots, p_{n}>0$ with $\sum_{k=1}^{n} p_{k}=1$. Then

Theorem 2-(i) (or Corollary 2) implies that $t \rightarrow \exp _{[t]}\left(\sum_{k=1}^{n} p_{k} \log _{[t]} x_{k}\right)$ is strictly monotone increasing on $[0,1]$. Note that $\exp _{[0]}\left(\sum_{k=1}^{n} p_{k} \log _{[0]} x_{k}\right)=\prod_{k=1}^{n} x_{k}^{p_{k}}$ and $\exp _{[1]}\left(\sum_{k=1}^{n} p_{k} \log _{[1]} x_{k}\right)=\sum_{k=1}^{n} p_{k} x_{k}$. Therefore, we obtain that

$$
\prod_{k=1}^{n} x_{k}^{p_{k}} \leq \exp _{[t]}\left(\sum_{k=1}^{n} p_{k} \log _{[t]} x_{k}\right) \leq \sum_{k=1}^{n} p_{k} x_{k}(0 \leq t \leq 1) .
$$

This is a new refinement of geometric-arithmetic mean inequality (cf. [9]).

\section{Acknowledgements}

The authors are grateful to the referee, for the careful reading of the paper and for the helpful suggestions and comments. Also, we would like to thank Professor Masatoshi Fujii for his helpful informations of $\nabla$ and Specht's ratio. S.-E. Takahasi is partially supported by Grant-in-Aid for Scientific Research, Japan Society for the Promotion of Science.

\section{Author details}

${ }^{1}$ The Open University of Japan, Chiba 261-8586, Japan ${ }^{2}$ Toho University, Yamagata University (Professor Emeritus), Chiba 273-0866, Japan

\section{Authors' contributions}

YN carried out the design of the study and performed the analysis. KK conceived of the study, and participated in its design and coordination. ST participated in the sequence alignment and drafted the manuscript. All authors read and approved the final manuscript.

\section{Competing interests}

The authors declare that they have no competing interests.

Received: 23 November 2010 Accepted: 5 September 2011 Published: 5 September 2011

\section{References}

1. Fujii, Jl, Fujii, M, Miura, T, Takagi, H, Takahasi, S-E: Continuously differentiable means. J Inequal Appl 2006, 15 (2006). (Art. ID75941)

2. Hewitt, E, Stromberg, K: Real and Abstract Analysis. Springer, Berlin (1969)

3. Long, BY, Chu, YM: Optimal inequalities for generalized logarithmic, arithmetic, and geometric means. J Inequal Appl 2010, 10 (2010). (Article ID 806825)

4. Matejicka, L: Proof of one optimal inequality for generalized logarithmic, arithmetic, and geometric means. J Inequal Appl 2010, 5 (2010). (Article ID 902432)

5. Kantorovich, LV: Functional analysis and applied mathematics. Uspechi Mat Nauk 3, 89-185 (1948). (in Russian)

6. Ptak, V: The Kantorovich inequality. Am Math Month. 102, 820-821 (1995). doi:10.2307/2974512

7. Tsukada, M, Takahasi, S-E: The best possiblity of the bound for the Kantorovich inequality and some remarks. J Inequal Appl. 1, 327-334 (1997). doi:10.1155/S1025583497000222

8. Fujii, M, Tominaga, M: An estimate of Young type operator inequality and Specht ratio RIMS Kôkyûroku. 1259, 173-178 (2002) (in Japanese)

9. Hara, T, Uchiyama, M, Takahasi, S-E: A refinement of various mean inequalities. J Inequal Appl. 2, 387-395 (1998). doi:10.1155/S1025583498000253

doi:10.1186/1029-242X-2011-48

Cite this article as: Nakasuji et al:: A new interpretation of Jensen's inequality and geometric properties of $\phi$-means. Journal of Inequalities and Applications 2011 2011:48. 